\title{
Application of a General Gas Electrode Model to Ni-YSZ Symmetric Cells: Humidity and Current Collector Effects
}

\author{
Eui-Chol Shin", Pyung-An Ahn", Hyun-Ho Seo ${ }^{\S}$, and Jong-Sook Lee ${ }^{\dagger}$ \\ School of Materials Science and Engineering, Chonnam National University, Gwangju 61186, Korea \\ (Received September 4, 2016; Revised September 17, 2016; Accepted September 19, 2016)
}

\begin{abstract}
Electrolyte-supported symmetric Ni-YSZ cermet electrodes of ca. $23 \mu \mathrm{m}$ were prepared by screenprinting and the impedance was measured as a function of humidity from $2 \%$ to $90 \%$ balanced in $\mathrm{H}_{2}$ at a total flow rate of $50 \mathrm{sccm}$. The Ni felt current collector of $1 \mathrm{~mm}$ thickness exhibited a Gerischer-like gas concentration impedance in the low frequency range, which was similarly observed in the cermet-supported solid oxide cells, while the Pt paste collector exhibited only electrochemical polarization. The electrochemical polarization of both samples was modeled by a non-ideal diffusion-reaction transmission line model including CPEs with $\alpha=0.5$. In the case of the Pt paste collector, all the Bisquert parameters exhibited humidity dependence to the $-1 / 2$ power, supporting a non-faradaic chemical reaction mechanism at three phase boundaries. Consequently, the surface diffusivity and reaction rate increased linearly with humidity. Less pronounced humidity dependence and somewhat lower utilization length with an Ni felt collector can be attributed to the diffusion-limited gas flow through the collector.
\end{abstract}

\section{Key words : Ni-YSZ cermet, Current collector, Gas concentration impedance, Humidity effects, General Bisquert model}

\section{Introduction}

I n this work, YSZ-supported symmetric cells with Ni-YSZ cermet electrodes were prepared and humidity effects on the gas electrode impedance were examined. Numerous studies of such symmetric cells have been reported. To correlate the microstructure and the electrochemical activity, 3-dimensional reconstruction using FIB-SEM has become popular recently. ${ }^{1-3)}$ For a mechanistic understanding, patterning of $\mathrm{Ni}$ electrodes has also been performed to correlate the electrochemical activity with the simplified microstructure. ${ }^{4-9)}$ Electrochemical characterization seems to have had relatively slow progress, however. Often, the gas concentration polarization and the electrochemical polarization are not properly distinguished.

Recently, the classical electrochemical model for a porous electrode in liquid electrolyte has been successfully applied to the Ni-YSZ and LSM-YSZ gas electrode reactions, in full Ni-YSZ/YSZ/LSM-YSZ solid oxide cells. ${ }^{10-12)}$ The classical electrode impedance in solution can be modeled as the transmission line in Fig. 1(a), with impedance

$$
Z_{B}=\left\{\frac{\chi_{1} \chi_{2}}{\chi_{1}+\chi_{2}}\left[\ell+\frac{2 \lambda}{\sinh (\ell / \lambda)}\right]+\lambda \frac{\chi_{1}^{2}+\chi_{2}^{2}}{\chi_{1}+\chi_{2}} \operatorname{coth}\left(\frac{\ell}{\lambda}\right)\right\}
$$

${ }^{\dagger}$ Corresponding author: Jong-Sook Lee

E-mail : jongsook@jnu.ac.kr

Tel : +82-62-530-1701 Fax : +82-62-530-1699 where $\ell$ is the thickness of the electrode layer and $\lambda=$ $\left.\sqrt{\zeta /\left(x_{1}+x_{2}\right)}\right)$. The distributed elements $x_{1}$ and $x_{2}$ (or $x_{2}$ and $x_{1}$ ) represent the impedance of the liquid paths and of the solid network, respectively; $\zeta$ represents the impedance of the solid/liquid interface. The model is available for fitting and simulation in a commercial program, Zview, as a Bisquert model (DX-11), notated as ' $B$ ', in the previous work, ${ }^{11,12)}$ where $x_{1}, x_{2}$, and $\zeta$ are each a parallel combination of a resistor $R$ and a CPE (constant phase element) with complex capacitance $Q^{*}=A(j \omega)^{\alpha-1}$. The model was probably first applied by Sonn et al. ${ }^{13)}$ to describe the electrochemical impedance on symmetrical cells with Ni-YSZ gas electrodes. It was employed for in-depth analysis of symmetric cells with Ni-CGO infiltrated cermets, ${ }^{14)}$ and also for analysis of the response assigned to Ni-YSZ electrodes in full cells. ${ }^{12)}$ Similarly, the model has been applied to symmetrical cells with LSM-ScYSZ, ${ }^{15)}$-CGO or -Ag-CGO electrodes, ${ }^{16)}$ and LSM-YSZ electrodes in full solid oxide cells. ${ }^{10-12)}$ The electrochemical behavior of LSM, essentially an electronic conductor, is comparable to that of $\mathrm{Pt}$ or Ni rather than to that of

This article is based on parts of a thesis for a doctoral degree of the first author: Eui-Chol Shin, Ph.D., Application of Transmission Line Impedance Models in Materials Research focusing on Energy Devices, Chonnam National University, 2015. The authors certify that there is no authorship dispute between them. "Presently at Samsung SDI Co., Ltd.

${ }^{\ddagger}$ Presently at Daewoo Compressor Co., Ltd.

${ }^{\S}$ Presently at Suwan Energy Co., Ltd. 
(a)

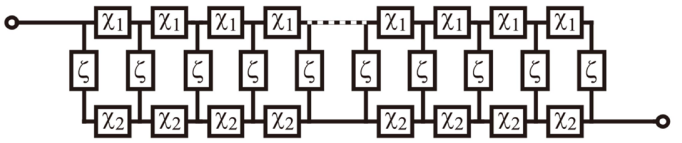

(b)

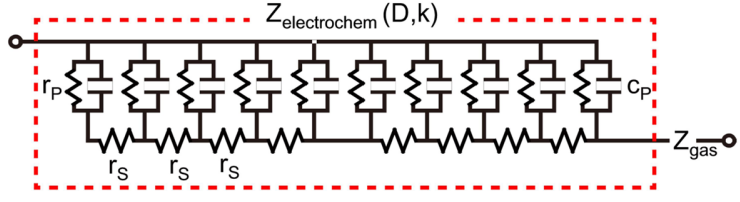

(c)

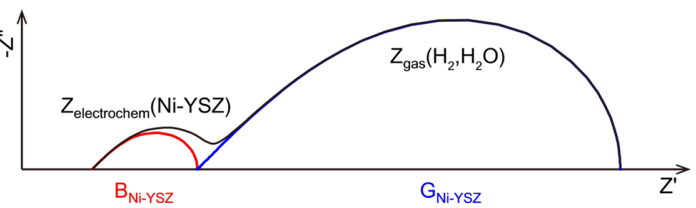

Fig. 1. (a) Generalized transmission line model for porous electrode or diffusion-recombination model, $B$, according to Bisquert notation. ${ }^{18,19)}$ (b) Impedance model for porous gas electrodes of solid oxide cells with predominantly electronic conductors such as $\mathrm{Pt}$, $\mathrm{Ni}$, or LSM in composites with ion conductors such as YSZ, CGO, etc. The electrochemical reaction is represented by $B$ with $r_{\mathrm{S}}, r_{\mathrm{p}}$ and $c_{\mathrm{p}}$ which is connected with the gas concentration impedance, $Z_{\text {gas }}$. (c) Hypothetical impedance spectra of Ni-YSZ cermet electrodes constructed based on previous report. ${ }^{12)}$

other mixed conducting perovskites.

In recent reports ${ }^{11,12)}$ by the present authors' group, unlike in other reports, ${ }^{13-16)}$ a simplified ideal three-parameter Bisquert model, as shown in Fig. 1(b), has been successfully applied. The resistance of the electronic path is considered negligible $\left(x_{1}=0\right)$, the ionic path is assumed to be purely resistive $\left(x_{2}=r_{\mathrm{S}}\right)$, and the interface reaction impedance $\zeta$ is modeled by a parallel circuit of $r_{\mathrm{P}}$ and $c_{\mathrm{P}}$. No CPE is used in the modelling. Then, the impedance of Eq. (1) can be simplified as

$$
Z_{\mathrm{B}}=\lambda r_{\mathrm{S}} \operatorname{coth}(\ell / \lambda)
$$

with the resistance magnitude $R_{\mathrm{B}}$ for $\omega \rightarrow 0$.

$$
R_{\mathrm{B}}=\sqrt{r_{S} r_{P}} \operatorname{coth}\left(\ell / \ell_{\delta}\right)
$$

where $\lambda=\left(\zeta / r_{\mathrm{S}}\right)^{1 / 2}, \zeta=r_{\mathrm{p}} /\left(1+j \omega r_{\mathrm{p}} c_{\mathrm{P}}\right)$, and the utilization length $\ell_{\delta}=\sqrt{\mathrm{r}_{\mathrm{P}} / \mathrm{r}_{\mathrm{S}}}$. The modeling allows a straightforward application of the diffusion-reaction kinetic model where (surface) diffusivity is derived as $D=\left(r_{\mathrm{S}} c_{\mathrm{P}}\right)^{-1}$, and (surface adsorption) reaction constant as $k=\left(r_{\mathrm{P}} c_{\mathrm{P}}\right)^{-1}$.

Although the two-rail transmission line model in Fig. 1(a) and Eq. (1) was suggested earlier by Levie ${ }^{17)}$ for electrochemical cells with liquid electrolytes, the recent attention associated with the diffusion-reaction kinetics is indebted to Bisquert and coworkers. ${ }^{18-22)}$ The impedance behavior of dye-sensitized solar cells (DSSCs) has also been gradually understood as a diffusion-(recombination) reaction process of the electrons in nanoporous semiconductor electrodes. ${ }^{18-22)}$
The diffusion-reaction kinetics of electrons can also be generally applicable to photovoltaic solar cells in the forward bias region. The interpretation of the transmission line model by diffusion-reaction kinetics for gas electrodes of solid oxide cells was recently disputed. ${ }^{16)}$ According to the arguments, transport in the ionic and electronic conducting paths should be driven by the electric field (drift), not by diffusion, and the interfacial resistance due to charge-transfer reaction, not by the interfacial reaction, i.e. as in the original model of liquid state electrochemistry. Although chargetransport and charge-transfer steps are certainly involved in the electrode kinetics of any electrochemical cell, these steps may not be the rate-controlling processes in solid-state electrochemical devices and thus may not explain the observed polarization impedance of solid oxide cells. This point has been emphasized by Prof. Mizusaki and coworkers over the decades. ${ }^{423-27)}$ Recently, a review has been published ${ }^{28)}$ in which these issues are, probably for first time, directly and explicitly addressed.

While the electrochemical impedance of Ni-YSZ electrodes in full solid oxide cells is often small compared to the other impedance components, and is severely affected by high frequency stray effects, the gas concentration impedance originating from Ni-YSZ cermet support in the low frequency region has been shown to be the major loss component for the low-humidity high-temperature operation of solid oxide cells. ${ }^{10,11)}$ Fig. 1(c) shows a simulated response of the hydrogen electrode based on the full cell results. ${ }^{11)} \mathrm{A}$ focus on humidity effects has been made in view of the high temperature electrolysis application of solid oxide cells and a dramatic variation of the gas concentration polarization of $\mathrm{Ni}$ YSZ cermet electrodes, $G_{\mathrm{Ni} \text {-YSZ, }}$, due to ambient and biasinduced humidity has been observed. ${ }^{10,11)}$ The gas concentration impedance of Ni-YSZ cermet supported fuel electrodes was more or less satisfactorily modeled as an ideal Gerischer response, ${ }^{10-12)}$ i.e.

$$
Z_{G}=\frac{R_{G}}{\sqrt{1+j \omega \tau G}}
$$

where $R_{\mathrm{G}}$ is the resistance magnitude. It should be noted that the Gerischer model is an infinite length version of the Bisquert model, in which the three parameters $r_{\mathrm{S}}, r_{\mathrm{P}}$, and $c_{\mathrm{P}}$ are reduced to two parameters $R_{\mathrm{G}}\left(=\left(r_{\mathrm{S}} r_{\mathrm{P}}\right)^{1 / 2}\right)$ and $\tau_{\mathrm{G}}\left(=\left(r_{\mathrm{P}} c_{\mathrm{P}}\right)\right) .{ }^{10,11)}$ There is no analytical model of the gas concentration impedance arising from gas flow dynamics. Numerical simulations showed a Warburg-like feature, ${ }^{29)}$ and the finite-length Warburg impedance model, modeled as an $r_{\mathrm{S}}{ }^{-} c_{\mathrm{P}}$ transmission line, has been used for the gas concentration impedance. ${ }^{13,30)}$ The response of the finite-length Warburg and Gerischer are, however, quite similar. ${ }^{10)}$ It has been suggested that a more generally applicable gas concentration impedance model, even a phenomenological one, can be a finite-length Gerischer or Bisquert model, ${ }^{11,12)}$ since at low velocity limits an ' $R C$ ' semi-circular gas concentration impedance results, similar to the 'reaction-limited' Bisquert model, in which $r_{\mathrm{P}}>r_{\mathrm{S}}{ }^{29)}$ 
Previously, the transmission line models, both for the electrochemical impedance in the Bisquert model and for the gas concentration impedance in the Gerischer model, were all employed in the ideal form with capacitors $c_{\mathrm{P} .}{ }^{11,12)} \mathrm{It}$ should be mentioned that the successful applications of the transmission line models in ideal form reported recently should not be considered to suggest or support that electrode responses indeed behave exactly according to the theoretical models. Rather, when transmission line models were employed in number four, the distributed nature of the ideal transmission line models was sufficient to reasonably describe the overlapped multiple responses of the full solid oxide cells. ${ }^{11,12)}$ Parameters derived therefrom are highly likely to be representative of the electrode reactions. Moreover, a priority may be given to the application of the ideal models, wherever possible, since the circuit parameters can be straightforwardly related to the kinetic parameters, as in the theory.

The purpose of this work is thus straightforward. The variation of the electrochemical kinetics of Ni-YSZ symmetric cells with humidity is to be examined by impedance spectroscopy. The impedance of the symmetric Ni-YSZ cells in the present work should appear in the ideal case as shown in Fig. 1(c). In addition to the gas concentration impedance, if any, only one Bisquert transmission line model should be used for the electrochemical polarization. It will be shown whether such an ideal model for Ni-YSZ electrodes can be applied at all, and how far parametric analysis of the physical significance can be performed when ideal models are not applicable.

\section{Experimental Procedure}

8 mol\% yttria-stabilized zirconia (YSZ) powder (TZ-8Y, Tosoh, Japan) was pressed into disk pellets by CIP at 147 $\mathrm{MPa}$ for $15 \mathrm{~min}$ and sintered at $1500^{\circ} \mathrm{C}$ for $2 \mathrm{~h}$. $\mathrm{NiO}(99.9 \%$ $\mathrm{BET}=2-4 \mathrm{~m}^{2} / \mathrm{g}, \mathrm{GCM}$, Korea) and the YSZ powders were mixed in a planetary ball mill (Pulverisette 6, Fritsch, Germany) for $4 \mathrm{~h}$. The ratio of NiO-YSZ powders to organic vehicles ( $\alpha$-terpineol of $94 \mathrm{wt} \%+$ ethyl cellulose $6 \mathrm{wt} \%$ ) was $1.5: 1$ and the paste was screen-printed on both sides of the YSZ disks and sintered for $2 \mathrm{~h}$ at $1400^{\circ} \mathrm{C}$. An Ni-YSZ layer of area ca. $0.2 \mathrm{~cm}^{2}$ was prepared using a screen printing mask of 200 mesh $(74 \mu \mathrm{m})$. Pt paste (Engelhard 6926, USA) and Ni felt (Sebang Global Battery, Korea) with pore size of $230 \mu \mathrm{m}$ and thickness of $1 \mathrm{~mm}$ were used, respectively, as current collectors. The thickness of the Ni-YSZ layer after sintering was ca. $23 \mu \mathrm{m}$. The cross-sectional microstructure of the cell with the Pt paste current collector on top is shown in Fig. 2. Disk-type cells were mounted on a lab-made solid oxide cell test unit (without sealing) at $850^{\circ} \mathrm{C}$; the gas flows were guided to flux on the tops of both surfaces. The details of the setup were reported elsewhere. ${ }^{31)}$ The humidity was controlled at a level up to $90 \%$ and was balanced with $\mathrm{H}_{2}$ using a custom-made direct evaporator (ADrop, GmbH, DV2MK-TL, Germany) based on a liquid flow meter

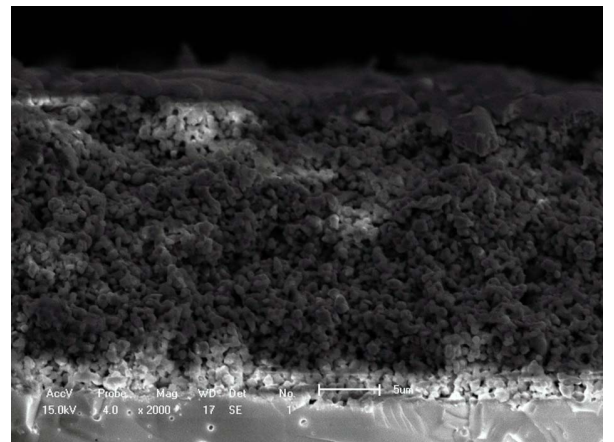

Fig. 2. SEM cross-section image of the Ni-YSZ/YSZ/Ni-YSZ half cell. The thin layer at the top is the Pt currentcollector.

(LIQUI-FLOW, Bronkhorst High-Tech B.V., The Netherlands). To avoid flow rate effects on the electrode kinetics, total gas flow rates were controlled at $50 \mathrm{sccm}$, as in the previous work. ${ }^{10,11)}$ The electrochemical measurements were performed on an Autolab PGSTAT 302N/FRA (Echo-Chemie B.V., The Netherlands) using the two-probe four-wire method. The applied frequency was in a range of $1 \mathrm{MHz}$ to $10 \mathrm{mHz}$, with an $\mathrm{AC}$ amplitude of $10 \mathrm{mV}$ rms. Impedance analysis and simulation were performed using the Zview program (Scribner Ass. Inc., USA).

\section{Results and Discussion}

The impedance spectra shown in Fig. 3 present the polarization behavior of the Ni-YSZ cermet electrodes under different humidity conditions for the two current collectors, $\mathrm{Pt}$ paste (left) and Ni felt (right). Although the Ni-YSZ electrodes were prepared in a similar way, the impedance characteristics are substantially different in terms of magnitude, shape, and humidity dependence. Based on the previous work, the impedance of the Ni-YSZ symmetrical cells can be modeled by the equivalent circuit shown in Fig. 4. In addition to the elements shown in Fig. 1(c), high frequency stray impedance, represented as $R_{\mathrm{STR}}$, is included in the model. As previously reported in detail, ${ }^{10)}$ not the inductor element $L$ but the addition of a resistance element $R_{\mathrm{STR}}$ in parallel reasonably describes the often-observed high frequency inductive loop behavior. This approach allows a systematic evaluation of the strongly overlapped multiple components, even though large errors are often inevitable.

The low frequency impedance component in the case of the Ni felt collector, shown in Fig. 3 (right), suggests the gas concentration impedance, $G_{\text {Ni-Ysz. }}$ in Fig. 1(c). It can be clearly seen in Fig. 3 (left) that the cell with the Pt paste collector for the similarly-prepared Ni-YSZ cermet electrodes does not display such an impedance component. Therefore, the behavior should be attributed to the $\mathrm{Ni}$ felt of $1 \mathrm{~mm}$ thickness used as a current collector. The Ni-mesh collector behaves in a manner similar to that of the porous thick cermet-support of state-of-the-art solid oxide cells in gas flow dynamics. The present results further support the idea that 

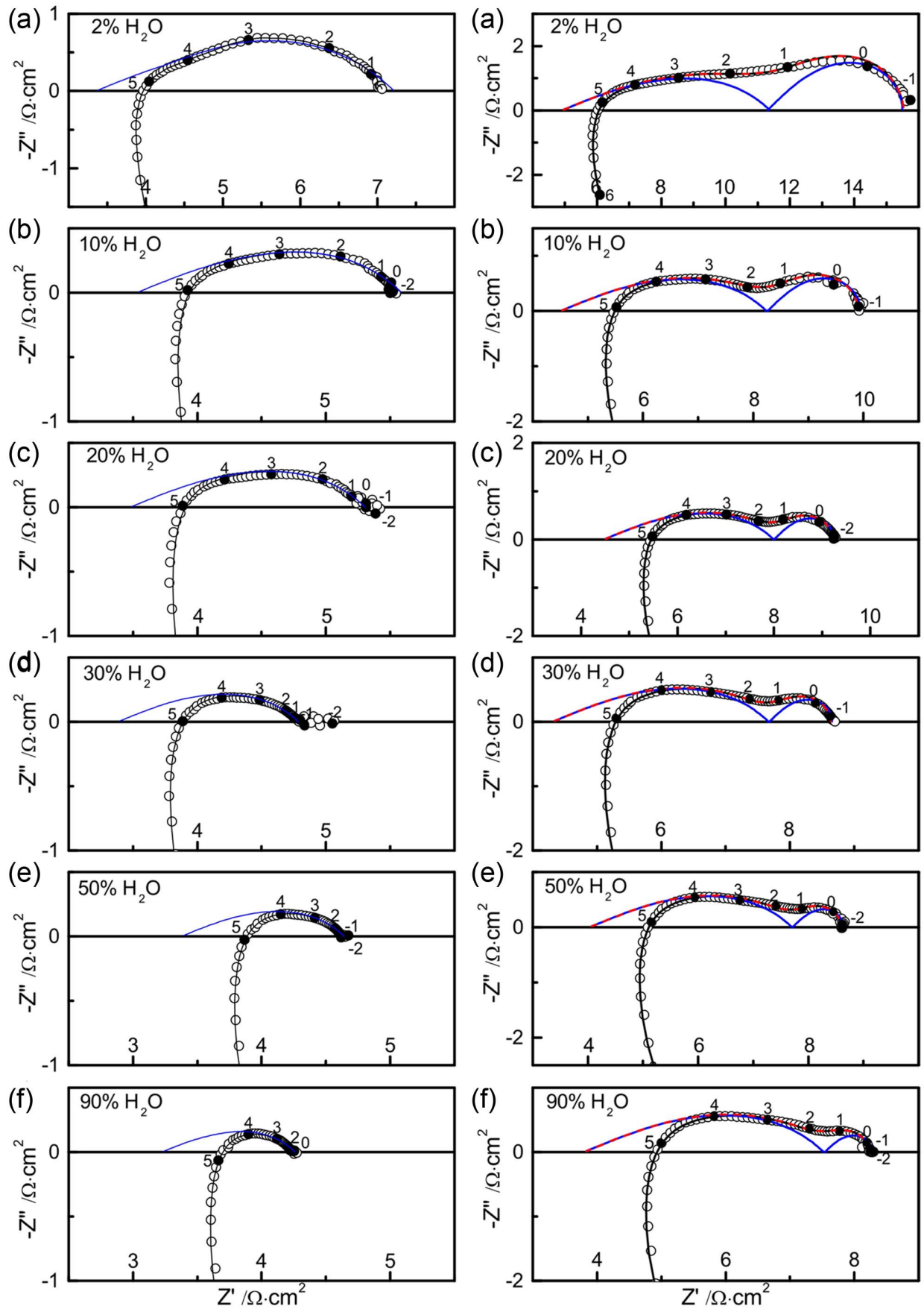

Fig. 3. Impedance spectra using Pt paste (left) and Ni felt (right) as current collectors at different humidity conditions. The blue solid lines and the red dashed lines in the impedance spectra are results simulated from fit results obtained using the equivalent circuit model shown in Fig. 4 without the stray impedance $L-R_{\mathrm{STR}}$.

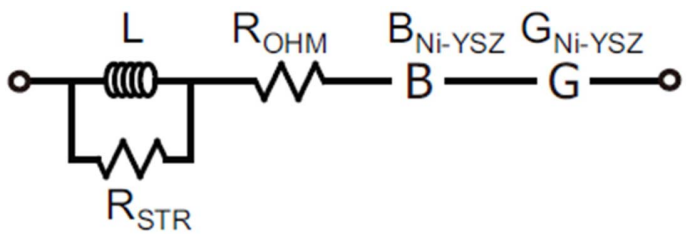

Fig. 4. Equivalent circuit model to fit impedance spectra of symmetric Ni-YSZ electrode cells, as shown in Fig. 3. the gas concentration impedance does not originate from the electrochemical reaction of the Ni-YSZ electrodes, which is expected to occur in common in both cells, but from the gas phase transport through the porous microstructure. ${ }^{10-12)}$ A similar origin may be supposed for the gas concentration impedance observed in the work of Sonn et al.., ${ }^{13)}$ in which $\mathrm{Ni}$-mesh current collectors were used for Ni-YSZ symmetric cells. It can be understood that the thin (less than a few $\mu \mathrm{m}$ 
thickness) Pt paste collector layer, as indicated in Fig. 2, does not cause any appreciable impedance in the gas phase transport through the layer.

Guillo et al. ${ }^{32)}$ compared different pastes of $\mathrm{Au}, \mathrm{Pt}$, and $\mathrm{Ni}$ and also Ni mesh as current collectors. In their work, $\mathrm{Ni}$ paste exhibited resistance far smaller than that of the other current collectors. Ni may be considered more catalytic than $\mathrm{Au}$ or Pt. Ni mesh, however, exhibited a response suggesting a Warburg- or Gerischer-like impedance, without any indication of the presence of a high frequency component for the given frequency range up to $10 \mathrm{kHz}$. In view of the present work, the Warburg- or Gerischer-like response of the Nimesh collector can be attributed to the gas concentration impedance. The other current collectors appear to exhibit mixed contributions, which depend on microstructural details as well as on the catalytic activities of the respective current collectors.

The true electrode reaction impedance of Ni-YSZ cermet electrodes is expected to be present in both cells, regardless of the current collectors. When the low frequency gas concentration impedance due to the thick Ni-mesh collector is considered for the response of the cell with the Ni felt collector, the presence of one similarly broad impedance response can be identified in the respective spectra for both cells; this can be ascribed to the electrochemical reaction of the $\mathrm{Ni}$ YSZ cermet electrodes.

Even when the strong overlapping of different components is taken into consideration, the broad electrochemical response of Ni-YSZ cermet electrodes in both cells cannot be described by an ideal Bisquert model, Eq. (2). The ideal Bisquert impedance is characterized by slope $1 / 2$ behavior in the high frequency region and a low frequency trajectory close to a semicircle, as can be seen in Fig. 1(c). For the experimental spectra shown in Fig. 3, the high frequency limit behavior is obscured by the stray impedance, and so a direct check for one half slope behavior is not possible. In the case of the Ni felt collector, the low frequency end of the electrochemical response also overlaps with the gas concentration impedance. Visible spectral features, however, are in any case too broad to be reasonably described by a single ideal Bisquert model. This situation is also generally true in many experimental results. Splitting the broad response into multiple components, possibly including one ideal Bisquert model seems not physically supported. In the measurement results shown in Fig. 3, little indication of multiple responses can be found.

Therefore, as in other reports ${ }^{13-16)} c_{\mathrm{P}}$ is generalized to nonideal capacitors or constant phase elements (CPEs) $q_{\mathrm{P}}$ with complex capacitance $q^{*}=a(j \omega)^{\alpha-1}$ with $\alpha$ less than 1 , as indicated in Fig. 5(a) in comparison to the scheme shown in Fig. 1(b). It should be noted that the equivalent circuit model now has four parameters $r_{\mathrm{S}}, r_{\mathrm{P}}, a_{\mathrm{p}}$, and $\alpha$. The coefficient $a_{\mathrm{P}}$ and the exponent $\alpha$, adjusted together, may give a good description of the experimental spectra. However, the coefficient and the exponent are likely to correlate with each other. The response of $\mathrm{CPE}$ with $\alpha<1$ extends over a wide frequency range, so the CPE parameters are in turn correlated with the parameters of the other components. Thus, the fit results with arbitrarily varying $a_{\mathrm{p}}$ and $\alpha$ are not expected to provide systematic information on the humidity effects or on the differences between the two collectors. As has been emphasized recently, ${ }^{33-36)}$ the employment of CPEs or other distributed elements with arbitrarily varying exponents of power-law frequency dependence should be avoided, if at all possible. Arbitrarily adjusted $a$ and $\alpha$ parameters are subject to strong correlation and thus introduce artefacts that can obscure behavior of physical significance, if any. It was also shown that the experimental data often directly indicate well-defined frequency dispersions so, when properly modeled, power-law exponents are welldefined constants that can be specific to certain physical mechanisms, to certain material systems, or to particular samples. These constants do not appear to arbitrarily vary with experimental conditions such as temperature, humidity, bias etc., for a given sample. Transmission line models with $q_{\mathrm{P}}$ describe so-called anomalous diffusion following fractional Fick's second law, in which the mean squared displacement is proportional to the fractional exponent $a$ of time. ${ }^{37)}$ The well-known 'generalized' Warburg impedance corresponds to the generalization of $c_{\mathrm{P}}$ to $q_{\mathrm{P}}$ in the $r_{\mathrm{S}} c_{\mathrm{P}}$ transmission line. For any physical significance in such kinetic mechanisms, $\alpha$ is not supposed to be an arbitrarily varying adjustable parameter.

When the spectra were fitted with the generalized Bisquert model, the fitted $\alpha$ values of the nonideal capacitors or the constant phase elements (CPEs) were found to be close to 0.5 , regardless of the humidity conditions for both cells. The modified transmission line model and a spectral feature with $\alpha=0.5$ are shown in Fig. $5(\mathrm{a}, \mathrm{b})$, which can be compared with the case of $\alpha=1$, shown in Fig. 1(b,c). It may be difficult to deduce the diffusion-reaction impedance characteristics in the strongly depressed arc with $\alpha=0.5$.

To compare the different collectors and different humidity conditions, $\alpha$ of $B_{\mathrm{Ni} \text {-YSZ }}$ was fixed at 0.5 for the final evaluation of the parameters reported in Figs. 6 and 7. The respective $B_{\mathrm{Ni}-Y S Z}$ (and the $G_{\mathrm{Ni}-\mathrm{YSZ}}$ component in the case of Ni felt

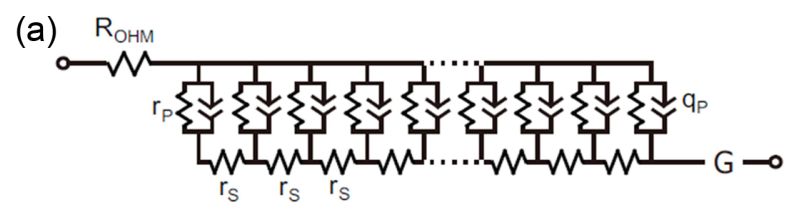

(b)

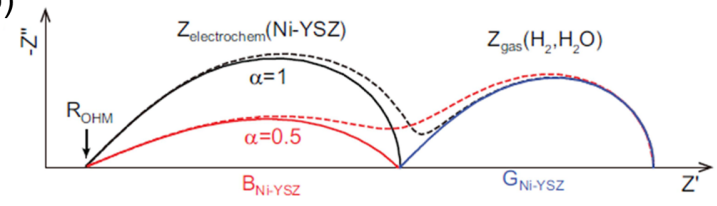

Fig. 5. (a) Bisquert transmission line model with $c_{\mathrm{P}}$ generalized to $q_{\mathrm{P}}$. (b) Hypothetical spectra with $\alpha=1$ and $\alpha=$ 0.5 in $B_{\mathrm{Ni}-\mathrm{YSZ}}$. 

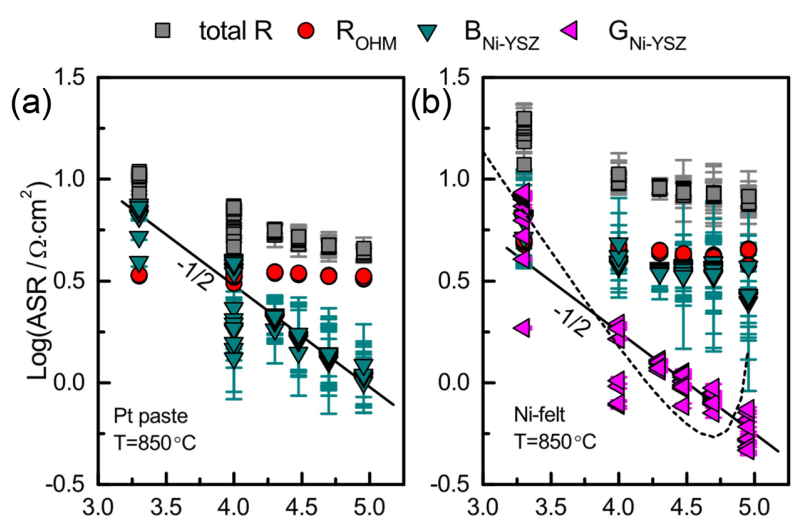

Fig. 6. Area specific resistance values of the respective impedance components of the equivalent circuit in Fig. 4 as a function of the humidity for the cells with $\mathrm{Pt}$ paste (a) and the Ni felt current collectors (b). The dashed curve in (b) represents the dependence of $G_{\mathrm{Ni} \text { YSI }}$ according to CSTR model.

collector) are simulated in blue lines in the spectra shown in Fig. 3. Fixed $\alpha$ may not describe the individual spectra best, but a slight variation in $\alpha$ can result in a seriously correlated evaluation of the other parameters and the trend of physical significance, if any, would be obscured. Conversely, if the evaluated parameters show a systematic trend of physical significance, the suggested model and its theoreti- cal background can be supported. Nonlinear least squares fitting analysis with the transmission line models as in Eqs. (1), (2), and (4), could be a critical hurdle for many experimental scientists. Fitting and simulation of the Bisquert and Gerischer model performed in this work were accomplished with the help of commercial software (Zview, Scribner Ass. USA).

In Fig. 6 the respective ASRs of the separated impedance components are represented as a function of the humidity in log-log plots for the cell with the Pt paste (a) and the Ni felt current collector (b), respectively. The humidity-independent ohmic contribution is deconvoluted as the high frequency intercepts of the simulated impedance spectra in Fig. 3, which are smaller than the experimental high frequency intercepts, which are conventionally taken to determine the ohmic contribution. For the Pt paste collector in Fig. 6(a), the rest of the electrode resistance corresponds to the electrochemical polarization modelled by one Bisquert impedance $B_{\mathrm{NiYSZ}}$ with $q_{\mathrm{P}}(\alpha=0.5)$, which is larger than the magnitude of the chord or the experimental arcs. The separated ASR of the general Bisquert element indicates humidity dependence close to $-1 / 2$ in the log-log plot. Multiple tests for the given humidity condition may be distinguished by error bars. In the estimation of $R_{\mathrm{B}}$ according to Eq. (3) errors are propagated from the errors in the fit parameters determined by a complex nonlinear least squares fitting. Large scattering in the data collected for the humidity level
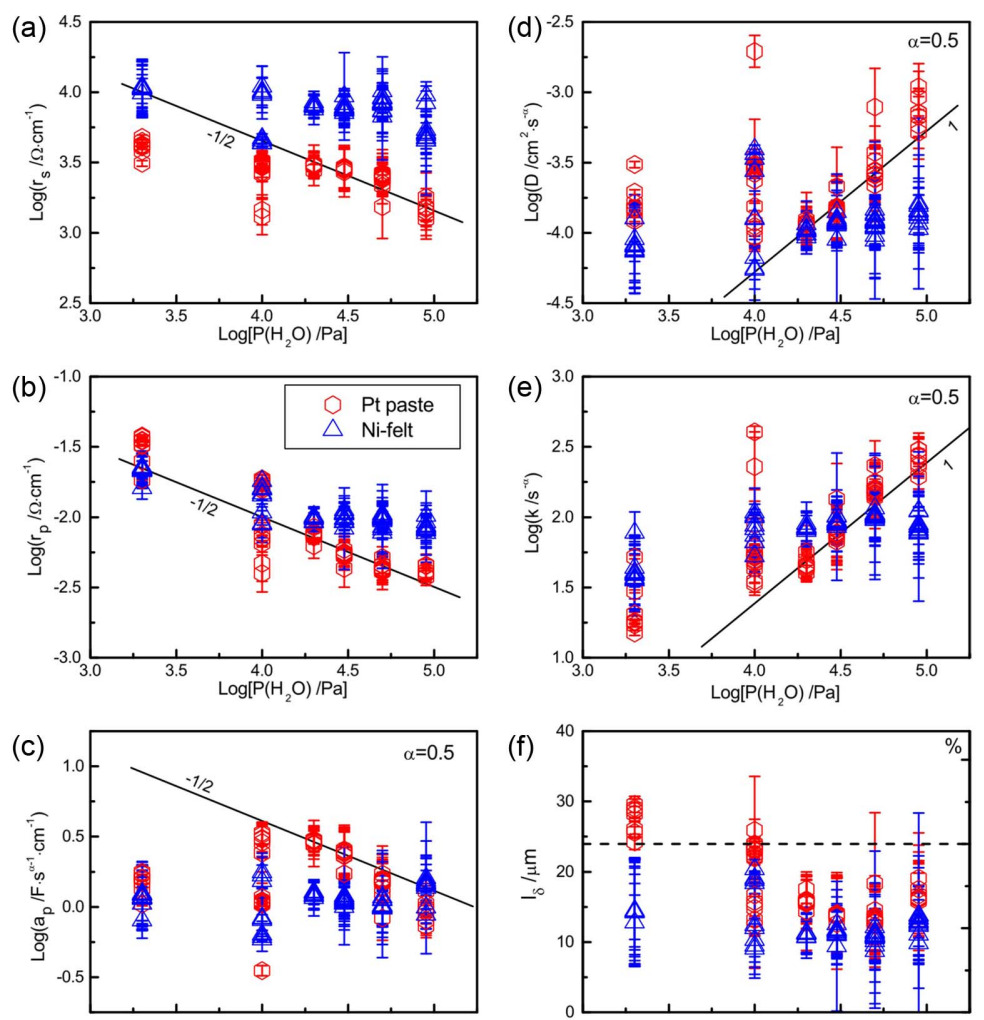

Fig. 7. Fit parameters of the polarization of Ni-YSZ cermet electrodes using a finite-length Gerischer element for $B_{\mathrm{Ni}-Y s Z}$ with ca. $\ell=23 \mu \mathrm{m} r_{\mathrm{S}}(\mathrm{a}), r_{\mathrm{P}}(\mathrm{b})$, and $a_{\mathrm{P}}(\mathrm{c})$, and the derived parameters $D(\mathrm{~d}), k(\mathrm{e})$, and $\ell_{\delta}(\mathrm{f})$ with Pt paste and Ni felt for current collector on the Ni-YSZ cermet electrode. 
of $10^{4} \mathrm{~Pa}$ and to a lesser degree for the level of $10^{3.3} \mathrm{~Pa}$ may be explained by the variation in the effective humidity in the respective measurements. The direct evaporator used for the humidity control in this work seems to fail to maintain a low humidity condition reliably.

The $\pm 1 / 2$ dependence on the gas phase activity can be generally explained by the exchange reaction of adsorbed species across the boundary of the two surfaces or along the edges or the three phase boundaries. ${ }^{27)}$ It should be noted that the ohmic resistance dominates the Bisquert contribution in high humidity condition, and thus total resistance decreases weakly in high humidity condition. Proper separation of the different components can thus be critical for a correct mechanistic understanding.

For the sample with the Ni felt collector, shown in Fig. 6(b), the gas concentration impedance, $G_{\mathrm{Ni} \text { YSZ }}$, suggests $-1 / 2$ humidity dependence, similar to the Bisquert impedance, $B_{\mathrm{Ni} \text {-YSZ }}$, in the case of the Pt paste collector shown in Fig. 6(a). Primdahl and Mogensen, ${ }^{38)}$ probably for the first time, definitely ascribed the low frequency response to 'gas conversion' impedance. Using a simple CSTR (continuously stirred tank reactor) model, this impedance was shown to be proportional to $\left(1 / x_{\mathrm{H}_{2} \mathrm{O}}+1 / x_{\mathrm{H}_{2}}\right){ }^{38,39)}$ which was also confirmed by the numerical simulations. ${ }^{29,40)}$ The dependence is shown as a dashed line in Fig. 6(b). Fig. 3(right) shows that the peak frequency values are more or less constant with humidity, indicating that the capacitance effects are inversely proportional to the resistance effects, as suggested. ${ }^{38)}$ As discussed previously, ${ }^{11)}$ gas concentration impedance, studied in depth, ${ }^{29,38-40)}$ considers an open space or chamber over a thin electrode layer. The gas concentration impedance through the thick porous cermet microstructure has not been theoretically addressed yet.

The electrochemical polarization component can also be described using Bisquert model $B_{\mathrm{Ni} \text { YSz }}$ with $\alpha=0.5$ for both Pt-paste and Ni felt collectors. In contrast to the behavior of the cell with the Pt-paste collector, Fig. 6(a), the magnitude of $B_{\mathrm{Ni} \text {-YSZ }}$ for the cell with the Ni felt collector decreases only slightly with humidity, as can be seen in Fig. $6(\mathrm{~b}) . B_{\mathrm{Ni} \text { YSZ }}$ is larger than $G_{\mathrm{Ni}-Y \mathrm{YZ}}$ except in the driest condition, and thus explains the weak humidity dependence of the total ASR. Primdahl and Mogensen ${ }^{38)}$ also reported that high frequency polarization decreased rather weakly with humidity compared to the low frequency gas concentration impedance, which appears consistent with the present observation. The humidity-independent electrochemical polarization is suggested to be correlated with the presence of gas concentration impedance. The gas concentration gradient imposed across the collector layer implies that the gas activity in the active Ni-YSZ layer of the inner region should be different from that in the layer surrounding the cell. The humidity level in the thin active Ni-YSZ layer may remain low even when the humidity in the outer region of the Ni felt current collector is high. This explains why the value of $B_{\mathrm{Ni} \text { YSz }}$ of the cell with the Ni felt collector remains similar to that of the cell with the Pt collector in low humidity conditions, as shown in Fig. 6.

In this work, similar to recent reports, ${ }^{10-12)}$ the behavior of the Bisquert transmission model parameters $r_{\mathrm{S}}, r_{\mathrm{P}}$, and $c_{\mathrm{P}}$ (in the present work $a_{\mathrm{p}}$ with $\alpha$ fixed at 0.5), is further examined. The Bisquert parameters and the derived kinetic parameters are presented in log-log plots in Fig. 7. For comparison, all the logarithmic scale ordinates indicate the variation over two orders of magnitude. It can be seen that the difference in $B_{\mathrm{Ni} \text { YSZ }}$ between the two collectors shown in Fig. 6, according to Eq. (3), is reflected in the parameter $r_{\mathrm{S}}$, as can be seen in Fig. 7(a). The magnitudes of the $r_{\mathrm{p}}$ parameters are closer, as can be seen in Fig. 7(b). When the four high humidity values with less variation are mainly considered, stronger humidity dependence is indicated in both the $r_{\mathrm{S}}$ and $r_{\mathrm{P}}$ parameters for the Pt paste collector roughly of $-1 / 2$ in log-log scale, in accordance with the behavior of $B_{\mathrm{Ni} \text {-YSZ }}$ in the dependence $-1 / 2$, as can be seen in Fig. 6(a) and as evaluated according to Eq. (3).

As discussed in the previous report, ${ }^{12)}$ the $r_{\mathrm{S}}$ parameter can exhibit a large fluctuation because it depends on the local connectivity. On the other hand, the $r_{\mathrm{P}}$ and $c_{\mathrm{P}}$ parameters for reaction resistance and adsorption capacitance, are expected to be less dependent on the connectivity. When $r_{\mathrm{S}}$ and $r_{\mathrm{P}}$, shown in Fig. 7(a) and (b) are compared, a larger scattering of $r_{\mathrm{S}}$ values than that for $r_{\mathrm{P}}$ can be clearly seen. The interpretation of $a_{\mathrm{p}}$ in Fig. 7(c) is not as straightforward as that of $c_{\mathrm{P}}$, however. In the present work, with $\alpha=0.5$, the $q_{\mathrm{p}}$ CPEs have a real admittance contribution of the same magnitude as the real capacitance. The $\alpha_{\mathrm{p}}$ parameters may be more affected by the connectivity than $c_{\mathrm{P}}$ parameters. The results for $a_{\mathrm{p}}$, shown in Fig. 7(c), compared to $r_{\mathrm{S}}$ in Fig. 7(a), strongly indicate the correlation between the two parameters. The data sets for the respective humidity condition are shown to exhibit similar scattering behavior. For Pt-paste collector, the two highest levels of humidity data are smaller than the $-1 / 2$ trend extrapolated from the two next highest sets of humidity data. For the case of the Ni felt collector, $a_{\mathrm{p}}$ values are humidity-independent. These values are comparable to the high humidity values in the case of the $\mathrm{Pt}$ paste collector.

The characteristics of the Bisquert parameters are directly carried over to the derived kinetic parameters of $D$ and $k$. For the ideal Bisquert model with $c_{\mathrm{P}}$ the parameters are derived as $D=\left(r_{\mathrm{S}} c_{\mathrm{P}}\right)^{-1}$ and $k=\left(r_{\mathrm{P}} c_{\mathrm{P}}\right)^{-1}$, in units of $\mathrm{cm}^{2} \cdot \mathrm{s}^{-1}$ and $\mathrm{s}^{-1}$, respectively. In the present work, $D=\left(r_{\mathrm{S}} a_{\mathrm{p}}\right)^{-1}$ and $k$ $=\left(r_{\mathrm{P}} a_{\mathrm{P}}\right)^{-1}$ in units of $\mathrm{cm}^{2} \cdot \mathrm{s}^{-\alpha}$ and $\mathrm{s}^{-\alpha}$, with $\alpha=0.5$ (Fig. 7(c,d)). In addition to the concept of anomalous diffusion in units of fractional exponents of time, as discussed above, the reaction rate constant derived here has units of fractional exponent of time. Normal units of the diffusivity and rate constants may be derived from an effective $C$ value using the relaxation time $\tau=(R A)^{1 / \alpha}$ as the representative value for $R C$ responses. ${ }^{21,41,42)}$ Such approaches are not, however, rigorously supported.

In the present work, the parameters $D$ and $k$ with exponents of time in fractional units can be compared between 
two different collectors since the same $\alpha$ value of 0.5 is involved. While $D$ and $k$ are humidity independent for the cell with the Ni felt collector, for the cell with Pt paste collectors the values of $D$ and $k$ are shown to approximately follow slope one dependence. The stronger increase and larger scattering in the two highest humidity values can be ascribed to the behavior of $a_{\mathrm{p}}$ in Fig. 7(c). Due to the opposite behavior in magnitude between $r_{\mathrm{S}}$ or $r_{\mathrm{p}}$ and $\alpha_{\mathrm{p}}$, the absolute magnitudes of $D$ and $k$ become comparable. The results may be considered to indicate intrinsic Ni-YSZ electrode properties that are common in both cells. The diffusion length or utilization length can be estimated as $\ell_{\delta}=\sqrt{r_{\mathrm{P}} / r_{S}}=\sqrt{D / k}$, and is presented in Fig. 7(f). The diffusion length is shown to be somewhat larger for the Pt paste collector; it becomes comparable to the layer thickness at low humidity conditions. For high humidity conditions, $\ell_{\delta} / \ell$ is constant at around $70 \%$. For Ni felt collectors it remains around 50\%. It appears consistent with the fact that a smaller $a_{\mathrm{p}}$ for the $\mathrm{Ni}$ felt collector is found than for the Pt-paste collector, as shown in Fig. 7(c). Smaller penetration length, smaller capacitance, and the weak humidity dependence in the case of the Ni felt collector can be related to the presence of gas concentration polarization resulting from the collector thickness of the Ni felt collector. With a gas concentration gradient imposed across the collector layer, the humidity surrounding the gas electrodes cannot be directly transferred to the active Ni-YSZ layer. Therefore, in low humidity conditions, the electrochemical polarization remains similar to that of the cell with the Pt paste collector.

\section{Conclusions}

The electrochemical polarization of Ni-YSZ cermet electrodes prepared as symmetric cells was investigated by impedance spectroscopy as a function of humidity. The cell response differs greatly depending on the current collectors, Pt paste or Ni felt. Only the cell with the Ni felt current collector exhibits Gerischer-like gas concentration impedance in the low frequency range, and thus confirms its flow dynamic origin, as in the response of cermet-supported state-of-the-art solid oxide cells. The high frequency response of the cell with the $\mathrm{Ni}$ felt collector and the overall response of the cell with the Pt paste collector are ascribed to the common electrochemical polarization of the Ni-YSZ electrodes. The response of the cell with the Pt-paste collector and the rest of the response of the cell with the $\mathrm{Ni}$ felt collector, when gas concentration contribution are subtracted, are similarly described by a high frequency stray impedance modeled as an $L R$ loop, a humidity-independent ohmic contribution, and a generalized Bisquert transmission line model for the surface diffusion-reaction co-limited electrochemical process in which $c_{\mathrm{P}}$ is generalized to $q_{\mathrm{P}} \mathrm{CPE}$ with $\alpha=0.5$. When the humidity-independent ohmic contribution is properly subtracted, a humidity dependence of -1 / 2 of the Bisquert parameters of $r_{\mathrm{S}}, r_{\mathrm{P}}$, and $a_{\mathrm{P}}$, as well as the polarization magnitude $B_{\mathrm{Ni}-\mathrm{YSZ}}$ and the +1 dependence of the derived kinetic parameters $D$ and $k$ are obtained for the Ptpaste collector. The results suggest that electrode polarization must be rate-limited by the non-faradaic chemical reaction at the triple phase boundaries. The weak humidity dependence of the Bisquert element in the case of the Ni felt collector can be related to the occurrence of gas concentration impedance $G_{\mathrm{Ni}-\mathrm{YSZ}}$, blocking the direct transfer of the surrounding gas activity to the active Ni-YSZ electrodes.

\section{Acknowledgments}

This research was performed for the Hydrogen Energy R\&D Center, one of the 21st Century Frontier R\&D Programs, funded by the Ministry of Science and Technology of Korea. This work was also partly supported by the World Class University (WCU) program (R32-2009-000-20074-0) and by the National Research Foundation of Korea (NRF) grant funded by the Ministry of Science, ICT \& Future Planning (NRF-2014R1A2A2A04004950).

\section{REFERENCES}

1. J. R. Wilson, W. Kobsiriphat, R. Mendoza, H.-Y. Chen, J. M. Hiller, D. J. Miller, K. Thornton, P. W. Voorhees, S. B. Adler, and S. A. Barnett, "Three-Dimensional Reconstruction of a Solid-Oxide Fuel-Cell Anode," Nat. Mater., 5 [7] 541-44 (2006).

2. P. Shearing, J. Golbert, R. Chater, and N. Brandon, "3D Reconstruction of SOFC Anodes Using a Focused Ion Beam Lift-Out Technique," Chem. Eng. Sci., 64 [17] 3928-33 (2009).

3. H. Iwai, N. Shikazono, T. Matsui, H. Teshima, M. Kishimoto, R. Kishida, D. Hayashi, K. Matsuzaki, D. Kanno, M. Saito, H. Muroyama, K. Eguchi, N. Kasagi, and H. Yoshida, "Quantification of SOFC Anode Microstructure Based on Dual Beam FIB-SEM Technique," J. Power Sources, 195 [4] 955-61 (2010).

4. J. Mizusaki, H. Tagawa, T. Saito, K. Kamitani, T. Yamamura, K. Hirano, S. Ehara, T. Takagi, T. Hikita, M. Ippommatsu, S. Nakagawa, and K. Hashimoto, "Preparation of Nickel Pattern Electrodes on YSZ and Their Electrochemical Properties in $\mathrm{H}_{2}-\mathrm{H}_{2} \mathrm{O}$ Atmospheres," J. Electrochem. Soc., 141 [8] 2129-34 (1994).

5. A. Utz, H. Stormer, A. Leonide, A. Weber, and E. Ivers-Tiff ee, "Degradation and Relaxation Effects of Ni Patterned Anodes in $\mathrm{H}_{2}-\mathrm{H}_{2} \mathrm{O}$ Atmosphere," J. Electrochem. Soc., 157 [6] B920-30 (2010).

6. W. G. Bessler, M. Vogler, H. Stormer, D. Gerthsen, A. Utz, A. Weber, and E. Ivers-Tiffée, "Model Anodes and Anode Models for Understanding the Mechanism of Hydrogen Oxidation in Solid Oxide Fuel Cells," Phys. Chem. Chem. Phys., 12 [42] 13888-903 (2010).

7. M. Vogler, A. Bieberle-Hutter, L. Gauckler, J. Warnatz, and W. G. Bessler, "Modelling Study of Surface Reactions, Diffusion, and Spillover at a Ni/YSZ Patterned Anode," J. Electrochem. Soc., 156 [5] B663-72 (2009).

8. A. M. Sukeshini, B. Habibzadeh, B. P. Becker, C. A. Stoltz, B. W. Eichhorn, and G. S. Jackson, "Electrochemical Oxi- 
dation of $\mathrm{H}_{2}, \mathrm{CO}$, and $\mathrm{CO} / \mathrm{H}_{2}$ Mixtures on Patterned Ni Anodes on YSZ Electrolytes,” J. Electrochem. Soc., 153 [4] A705-15 (2006).

9. M. Liu, M. E. Lynch, K. Blinn, F. M. Alamgir, and Y. Choi, "Rational SOFC Material Design: New Advances and Tools," Mater. Today, 14 [11] 534-46 (2011).

10. E.-C. Shin, P.-A. Ahn, H.-H. Seo, J.-M. Jo, S.-D. Kim, S.-K. Woo, J. H. Yu, J. Mizusaki, and J.-S. Lee, "Polarization Mechanism of High Temperature Electrolysis in a Ni-YSZ/ YSZ/LSM Solid Oxide Cell by Parametric Impedance Analysis," Solid State Ionics, 232 80-96 (2013).

11. E.-C. Shin, P.-A. Ahn, H.-H. Seo, D. T. Nguyen, S.-D. Kim, S.-K. Woo, J. H. Yu, and J.-S. Lee, "Pinning-down Polarization Losses and Electrode Kinetics in Cermet-Supported LSM Solid Oxide Cells in Reversible Operation," Solid State Ionics, 277 1-10 (2015).

12. E.-C. Shin, J. J. Ma, P.-A. Ahn, H.-H. Seo, D. T. Nguyen, and J.-S. Lee, "Deconvolution of Four Transmission-LineModel Impedances in Ni-YSZ/YSZ/LSM Solid Oxide Cells and Mechanistic Insights," Electrochim. Acta, 188 240-53 (2016).

13. V. Sonn, A. Leonide, and E. Ivers-Tiffée, "Combined Deconvolution and CNLS Fitting Approach Applied on the Impedance Response of Technical Ni/ 8YSZ Cermet Electrodes," J. Electrochem. Soc., 155 B675-79 (2008).

14. J. Nielsen, T. Klemens, and P. Blennow, "Detailed Impedance Characterization of a Well Performing and Durable Ni: CGO Infiltrated Cermet Anode for Metal-Supported Solid Oxide Fuel Cells," J. Power Sources, 219 305-16 (2012).

15. T. Ramos, M. Søgaard, and M. B. Mogensen, "Electrochemical Characterization of Ni/ScYSZ Electrodes as SOFC Anodes," J. Electrochem. Soc., 161 [4] F434-44 (2014).

16. J. Nielsen and J. Hjelm, "Impedance of SOFC Electrodes: A Review and a Comprehensive Case Study on the Impedance of LSM: YSZ Cathodes," Electrochim. Acta, 115 31-45 (2014).

17. R. de Levie, "On Porous Electrodes in Electrolyte Solutions: I. Capacitance Effects," Electrochim. Acta, 8 [10] 751-80 (1963).

18. J. Bisquert, "Influence of the Boundaries in the Impedance of Porous Film Electrodes," Phys. Chem. Chem. Phys., 2 [18] 4185-92 (2000).

19. J. Bisquert, "Theory of the Impedance of Electron Diffusion and Recombination in a Thin Layer," J. Phys. Chem. B, 106 [2] 325-33 (2002).

20. F. Fabregat-Santiago, J. Bisquert, E. Palomares, L. Otero, D. Kuang, S. M. Zakeeruddin, and M. Gratzel, "Correlation between Photovoltaic Performance and Impedance Spectroscopy of Dye- Sensitized Solar Cells Based on Ionic Liquids," J. Phys. Chem. C, 111 [17] 6550-60 (2007).

21. F. Fabregat-Santiago, G. Garcia-Belmonte, I. Mora-Sero, and J. Bisquert, "Characterization of Nanostructured Hybrid and Organic Solar Cells by Impedance Spectroscopy," Phys. Chem. Chem. Phys., 13 [20] 9083-118 (2011).

22. J. Bisquert, I. Mora-Sero, and F. Fabregat-Santiago, "Diffusion-Recombination Impedance Model for Solar Cells with Disorder and Nonlinear Recombination," ChemElectroChem, 1 [1] 289-96 (2014).
23. J. Mizusaki, K. Amano, S. Yamauchi, and K. Fueki, "Electrode Reaction at $\mathrm{Pt}, \mathrm{O}_{2}(\mathrm{~g}) /$ Stabilized Zirconia Interfaces. Part I: Theoretical Consideration of Reaction Model," Solid State Ionics, 22 [4] 313-22 (1987).

24. J. Mizusaki, K. Amano, S. Yamauchi, and K. Fueki, "Electrode Reaction at $\mathrm{Pt}, \mathrm{O}_{2}(\mathrm{~g}) /$ Stabilized Zirconia Interfaces. Part II: Electrochemical Measurements and Analysis," Solid State Ionics, 22 [4] 323-30 (1987).

25. J. Mizusaki and H. Tagawa, "Microstructure and Electrochemical Property relationship for $\mathrm{O}_{2}$ Reduction in Zirconia Cells," pp. 75-87 in Proc. of the Symposium on High Temperature Electrode Materials and Characterization. Eds. by D. D. MacDonald and A. C. Khandkar, Vol. 91-6. The Electrochem. Soc., Pennington, NJ, 1991.

26. J. Mizusaki, H. Tagawa, T. Saito, T. Yamamura, K. Kamitani, K. Hirano, S. Ehara, T. Takagi, T. Hikita, M. Ippommatsu, S. Nakagawa, and K. Hashimoto, "Kinetic Studies of the Reaction at the Nickel Pattern Electrode on YSZ in $\mathrm{H}_{2}-\mathrm{H}_{2} \mathrm{O}$ Atmospheres," Solid State Ionics, 70 52-8 (1994).

27. J. Mizusaki, H. Tagawa, K. Isobe, M. Tajika, I. Koshiro, H. Maruyama, and K. Hirano, "Kinetics of the Electrode Reaction at the $\mathrm{H}_{2}-\mathrm{H}_{2} \mathrm{O}$ Porous Pt/Stabilized Zirconia Interface," J. Electrochem. Soc., 141 [6] 1674-83 (1994).

28. J. Mizusaki, "Model for Solid Electrolyte Gas Electrode Reaction Kinetics; Key Concepts, Basic Model Construction, Extension of Models, New Experimental Techniques for Model Confirmation, and Future Prospects," Electrochemistry, 82 [10] 819-29 (2014).

29. W. Bessler, "Gas Concentration Impedance of Solid Oxide Fuel Cell Anodes I. Stagnation Point Flow Geometry," J. Electrochem. Soc., 153 A1492-54 (2006).

30. A. Leonide, B. Ruger, A. Weber, W. Meulenberg, and E. Ivers-Tiffee, "Impedance Study of Alternative (La,Sr) $\mathrm{FeO}_{3-\delta}$ and (La,Sr)(Co,Fe) $\mathrm{O}_{3-\delta}$ MIEC Cathode Compositions," J. Electrochem. Soc., 157 B234-39 (2010).

31. G.-R. Kim, H.-H. Seo, J.-M. Jo, E.-C. Shin, J. H. Yu, and J.S. Lee, "Moving Boundary Diffusion Problem for Hydration Kinetics Evidenced in Non-Monotonic Conductivity Relaxations of Proton Conducting Perovskites," Solid State Ionics, 272 60-73 (2015).

32. M. Guillodo, P. Vernoux, and J. Fouletier, "Electrochemical Properties of Ni-YSZ Cermet in Solid Oxide Fuel Cells: Effect of Current Collecting," Solid State Ionics, 127 [1] 99107 (2000).

33. J.-H. Kim, E.-C. Shin, D.-C. Cho, S. Kim, S. Lim, K. Yang, J. Beum, J. Kim, S. Yamaguchi, and J.-S. Lee, "Electrical Characterization of Polycrystalline Sodium $\beta "$ "Alumina: Revisited and Resolved," Solid State Ionics, 264 22-35 (2014).

34. S.-H. Moon, D.-C. Cho, D. T. Nguyen, E.-C. Shin, and J.-S. Lee, "A Comprehensive Treatment of Universal Dispersive Frequency Responses in Solid Electrolytes by Immittance Spectroscopy: Low Temperature AgI Case," J. Solid State Electrochem., 19 [8] 2457-64 (2015).

35. S.-H. Moon, Y. H. Kim, D.-C. Cho, E.-C. Shin, D. Lee, W. B. Im, and J.-S. Lee, "Sodium Ion Transport in Polymorphic Scandium NASICON Analog $\mathrm{Na}_{3} \mathrm{Sc}_{2}\left(\mathrm{PO}_{4}\right)_{3}$ with New Dielectric Spectroscopy Approach for Current-Constriction Effects," Solid State Ionics, 289 55-71 (2016). 
36. J.-S. Lee, "A Superior Description of AC Behavior in Polycrystalline Solid Electrolytes with Current-Constriction Effects,” J. Korean Ceram. Soc., 53 [2] 150-61 (2016).

37. J. Bisquert and A. Compte, "Theory of the Electrochemical Impedance of Anomalous Diffusion," J. Electroanal. Chem., 499 [1] 112-20 (2001).

38. S. Primdahl and M. Mogensen, "Gas Conversion Impedance: A Test Geometry Effect in Characterization of Solid Oxide Fuel Cell Anodes," J. Electrochem. Soc., 145 [7] 2431-38 (1998).

39. S. Primdahl and M. Mogensen, "Gas Diffusion Impedance in Characterization of Solid Oxide Fuel Cell Anodes," J.
Electrochem. Soc., 146 2827-33 (1999).

40. W. Bessler and S. Gewies, "Gas Concentration Impedance of Solid Oxide Fuel Cell Anodes II. Channel Geometry," J. Electrochem. Soc., 154 B548-59 (2007).

41. P.-A. Ahn, E.-C. Shin, G.-R. Kim, and J.-S. Lee, "Application of Generalized Transmission Line Models to Mixed Ionic-Electronic Transport Phenomena," J. Korean Ceram. Soc. 48 [6] 549-58 (2011).

42. P.-A. Ahn, E.-C. Shin, J.-M. Jo, J. H. Yu, S.-K. Woo, and J.S. Lee, "Mixed Conduction in Ceramic Hydrogen/Steam Electrodes by Hebb-Wagner Polarization in the Frequency Domain," Fuel Cells, 12 1070-84 (2012). 